\title{
Optimization of the heat output of wall convectors with using an unconventional slumped glass cover
}

\author{
Petr Kulhavy ${ }^{1, a}$, Jitka Kleckova ${ }^{2}$, Michal Petru ${ }^{1}$ and Miroslav Havlicek ${ }^{3}$ \\ ${ }^{1}$ Faculty of Mechanical Engineering, Technical University of Liberec, Studentská 2, 461 17, Liberec 1, Czech Republic \\ ${ }^{2}$ Department of Mechanics, Faculty of Applied Sciences, University of West Bohemia, Pilsen. Univerzitní 22, Pilsen, Czech Republic. \\ ${ }^{3}$ Licon heat s.r.o., Construction department, Průmyslová zóna Sever, 460 11, Liberec 11, Czech Republic
}

\begin{abstract}
This paper deals with study of a new shape of the glass cover of heat convectors. Design of the heat convectors is characterized by the low water volume, highly variable geometry, adjustable heat output and the pressure losses that arise when the fluid flow through the exchanger. Based on a new concept of a slumped glass cover of the exchanger have been created some numerical models and also carried out experiments in order to find and optimize an appropriate shape. The glass materials are very specific mainly due to their specific shaping abilities and technological capabilities of manufacturing. The aim is to determine an appropriate shape and a curvature of the glass body and also the position and size of the exchanger. It has been found a significant dependence of the heat exchanger position on to the total heat output of an entire device. Simultaneously has been proved also the dependence of a shape of the cover plate, to the total heat output, that is more considerably for natural than for the forced convection.
\end{abstract}

\section{Introduction}

For a heating of houses, flats and industrial buildings are being used a lot of various devices. From the still available and most commonly used conventional castiron radiators to devices, supplemented by regulation and fans, so-called convectors. Classic radiators are used for the transmission and transformation of heat into the surroundings through radiation. Modern heating bodies, called convectors use principle of free or forced convection, when the air movement is accelerated by the fan [1]. The energy that we can obtain is usually from the electricity, fossil fuels or woods. The final conversion of the supplied energy to thermal has been carried out using our own equipment or from heating plants, such as longhaul networks or central distribution systems. In most cases is the thus obtained energy further transmitted to a fluid medium in the mostly number of cases to water, sometimes also oils etc.. This way is the thermal energy distributed through buildings and by using exchangers, is the heat transferred into the rooms. Today there is possible to choose from an enormous offer of heating elements and their variants. Other possibilities including e.g. the floor heating systems and the current top, we can say, are the individual central distribution systems with heat pump, often complimented by recuperation or air conditioning units. These devices instead of only a

\footnotetext{
${ }^{\mathrm{a}}$ Corresponding author: petr.kulhavy@tul.cz
}

temperature control at the same time, providing adequate exchange, regulation of humidity and air quality. Practically in all industries, with regard to an efficient use of energies, we encounter reduction of the medium (water) volume, a total weight of the device and the input power. As mentioned earlier from the original cast iron radiators is the move over time towards their smaller, lighter and more powerful variants. Generally, heating convectors designed for the heating in the living spaces, have been gradually replacing the classic radiators $[1,2]$. The most important parameters indicating the competitiveness of products is definitely their thermal power and in the case of convectors with forced convection also an energy consumption and acoustic characteristics of the fans, namely the sound pressure level. In these parameters is already achieved almost a physical maximum and more important than a little bit exceed the others, is primarily to keep the pace with the top. Currently, a real fight is in this sector on the field of design. There are e.g. various micro lamellar systems [3], iron castings sculptures or other highly complicated shapes. Furthermore, for this sector completely new materials have been used like e.g. glass, mirror and polished stone or wooden board [4]. In our case we concentrated to a glass plate manufactured of technology 
so-called "slumping" with simultaneous usage of a fusing. The aim of this work is in cooperation with the manufacturer of heat convectors Licon Llc assess the dependency of a shape of the front cover plate and position of the heat exchanger on the overall thermal performance.

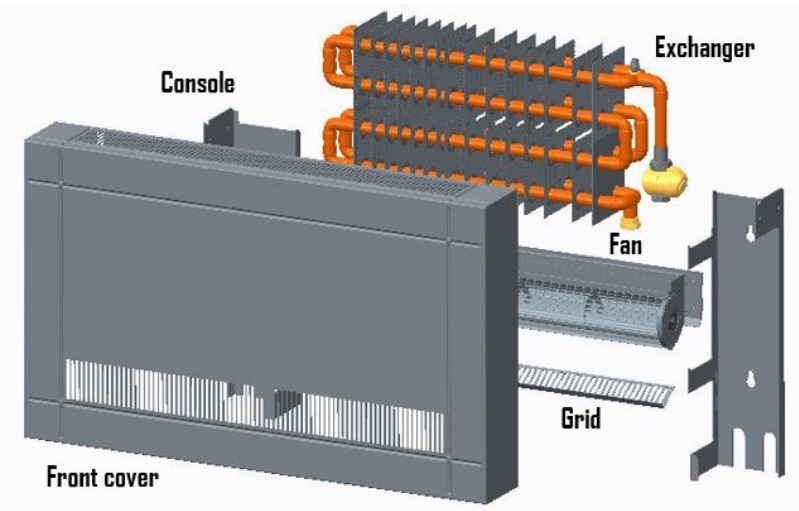

Figure 1. The original version of the wall-mounted convector.

\section{The solved problem}

About the advantages of devices based on convection instead of only radiation for heating in living rooms speak a lot of researchers. We can convince about it eg. at the work of Hamza [5], which speaks about energy savings in some model and simulated situations. This work is comparing two methods of heating (radiation, convection) in an experimental room at different ambient temperatures. A lot of international authors e.g. [6,7,8] deal with the question of an appropriate type of convection and they have been trying to find out a compromise between thermal comfort and the size of the supplied energy. The results showed that in energy terms is more advantageous to use the natural convection instead of the forced convection. This should be logically caused by the fact that the movement of warm air during heating arises naturally and there isn't need to supply any additional electric energy. Such a solution appears to be preferable, especially in terms of energy saving. The usage of the fan, the control and power required for the power supply, directly associate also with the end product and overhead cost [2,5]. From the other side, with the forced convection is generally reached significantly higher levels of heat output and faster achieved feeling of thermal comfort in the entire heated space and that is why this method is so widely preferred. The question and the subject of many numerical models and experiments, remains the subjective feeling and perception of thermal comfort. In our case the wall-mounted radiator, that standardly uses the forced convection, is tested and innovated. The volume of water in the individual types of used heat exchangers is in ranges from 0.18 to 1.2 liters per meter of length and the volumetric flow up to $51 / \mathrm{min}$. The thermal output, of the tested wall mounted convectors, according to EN 442 at an ambient temperature $20^{\circ} \mathrm{C}$ and at the operation mode $70 / 55^{\circ} \mathrm{C}$, reaches the values in range from approximately 190 to
$7800 \mathrm{~W}$ depending on length of the convector, flow rate and a revolutions of used fan.

\subsection{The tested prototype}

As is already mentioned above, the leading manufacturers of heating systems has already reached the real maximum of thermal performance and now is the competition especially in a field of design. In our case, the original steel body of convector (Fig.1) was replaced by a transparent front plate made from slumped glass (Fig. 2). This slumped cover is dominant not only for aesthetic reasons, but also due to a very specific shapes that can be achieved and also can be optimized with regard to the internal flow with the aim to increase heat output.

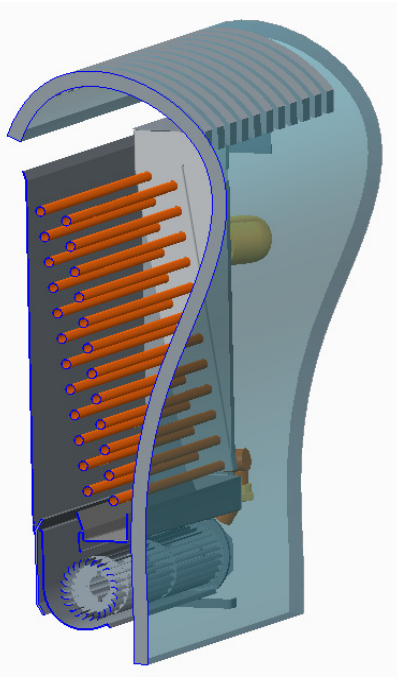

Figure 2. The first idea of a shape the slumped glass cover.

By research of an ideal shape of the fins fitted on tubes of a heat exchanger, their position and air flowing around them have dealt very thoroughly eg. Lemfeld and Frana $[2,9,10]$, as shown in Figure 3.
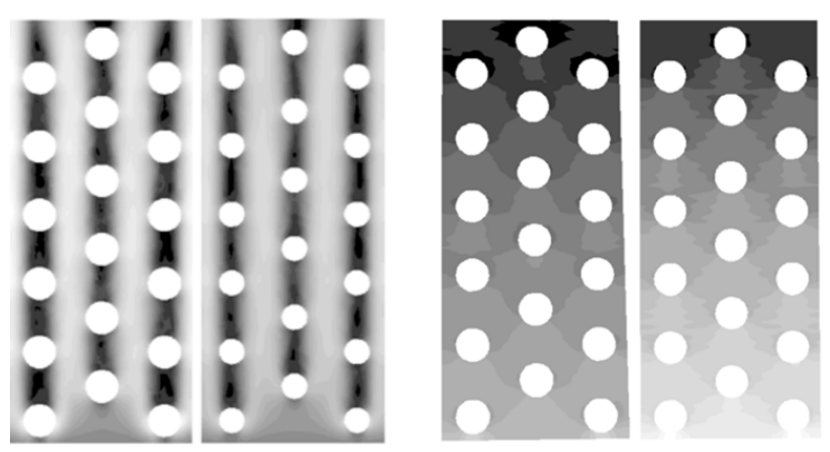

Figure 3. Example of distributions temperature fields (left) and static pressure (right) for different types of fins [9].

\section{Experiments}

Experiments were performed in thermodynamic laboratories of the manufacturer. The aim of the carried tests was to assess the effect of shape of the innovated cover to the thermal output and optimizing its geometry. 
The thermal power was measured using a thermocouple system. Measurements were also conducted for the exchanger without any sheathing. Instead of the glass was at first used transversely formable plastic material on the inner side covered with thermally insulating and reflective foils. With that is possible to achieve changeable geometric shapes. To achieve the exact shape and repeatability of the experiment, it was used steel templates, created in accordance to the CAD drawings. The desired final shape (Fig. 4) was with using a CNC machined mold made of a slumped glass.

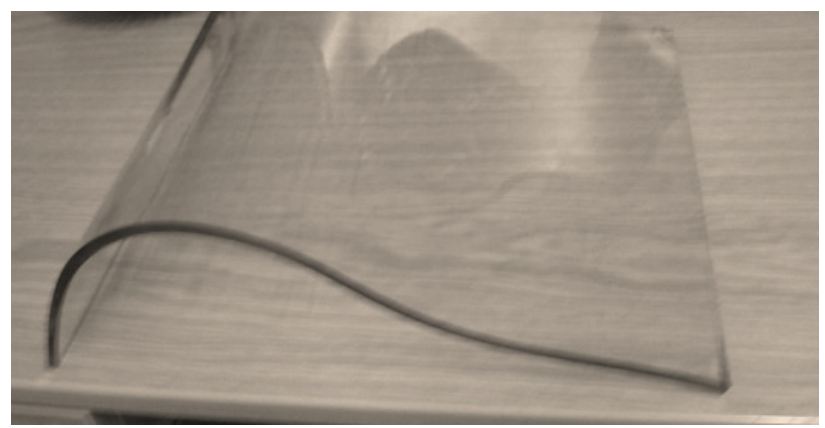

Figure 4. The first created prototype of a glass cover.

Wall convector completed by some changeable covering panels was connected to the water circuit in manufacturer's hydrodynamic laboratory. For testing liquid at a given temperature it is necessary to ensure a sufficiently powerful heating. It was used a thermally insulated tank with electric heating dimensioned over the maximum estimated thermal performance of the connected devices. Liquid pumped with an electronically controlled pump, fed from the tank through the control ultrasonic flowmeter into the exchanger of the tested wall convector and then is the liquid again returned to the tank [1]. The measurement was performed for 2-point temperature of water $65 / 55^{\circ} \mathrm{C}$ and the ambient temperature $20^{\circ} \mathrm{C}$. The electronically controlled variable flow rate in a range $0.1-51 \times \min -1$ was joined to the connecting fitting of the heat exchanger (Fig. 5). This constitutes a speed from approximately 0.01 to $0.3 \mathrm{~m} / \mathrm{s}$ when a size of the Re number reaches value 100-4800.

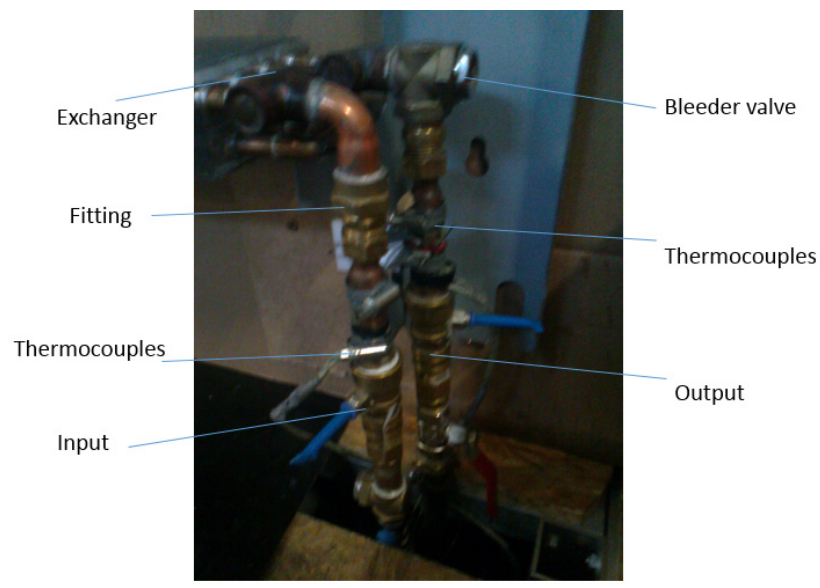

Figure 5. Joining parts of the exchanger with thermocouples.
Measurements were performed by using the interface National Instruments - NI cDAQ-9174 with additional modules. To the module are connected $\mathrm{NiCr}-\mathrm{Ni}$ thermocouples. Their installation is 3 to input, 3 to output and the final value is their average. Several other couples measure the temperature in the heater, the pump and of course the ambient temperature. Based on these data, is the density of water calculated for the precise determination of the mass flow.

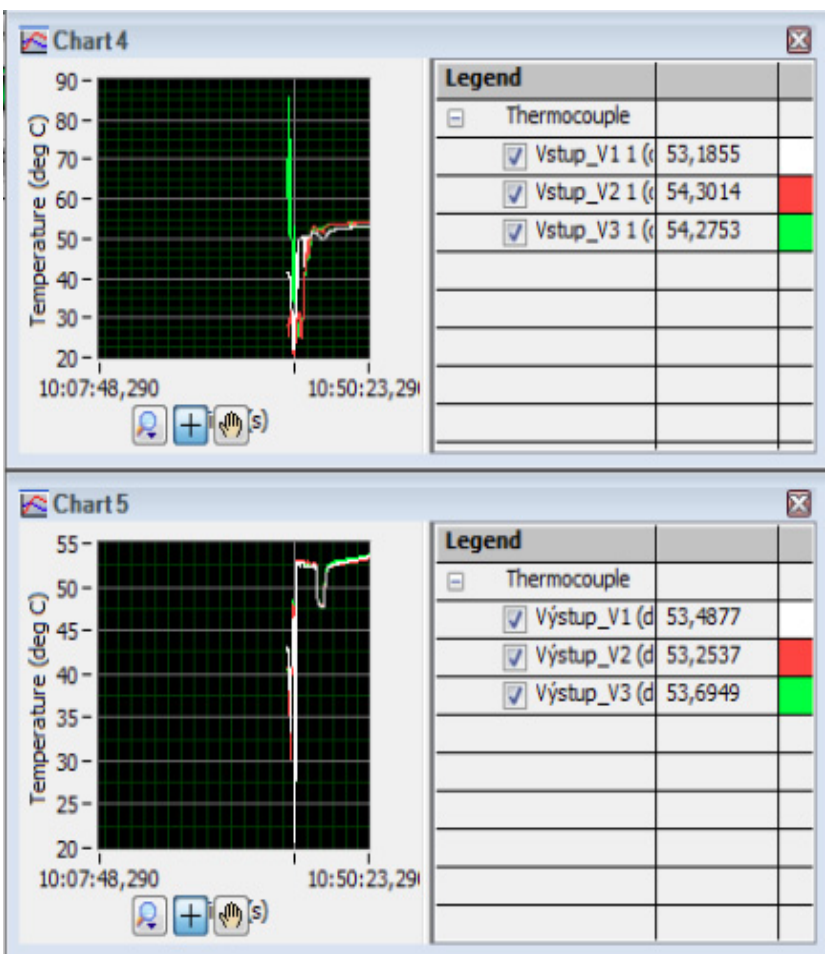

Figure 6. Interface of used $\mathrm{NI}^{\circledR}$ Lab View measurement software.

\section{Numerical simulation}

Experimental results were compared with numerical results obtained by using commercial CFD software ANSYS Fluent, which allows the modelling of flow of isotropic and anisotropic structures and other physical phenomenon. This software contains a wide variety of tools for simulation of the various problems, which are described by partial differential equations. CFD software ANSYS Fluent uses for the calculation the implicit algorithm, where individual states of the analyzed compression are updated gradually in time $t$ to time $t+d t$ according to Eq. (1).

$$
\delta u_{i+1}=u_{i+1}^{t+\Delta t}-u_{i}^{t+\Delta t}
$$

where $u_{i}^{t+\Delta t}$ is vector of nodal displacements for $i^{\text {th }}$ iteration in the time $t+\Delta t$.

\subsection{Numerical model}

Two cases of heat convectors were compared by using numerical simulations. The type with new glass cover and convector with standard plated cover. To simplify a 
problem, computational domain was considered as a twodimensional section of the original model, as shown in Fig. 7. The computational mesh was thickened in the vicinity of walls of heat exchanger tubes. Numerical simulations were accomplished for the temperature of tubes equal to $65^{\circ} \mathrm{C}$. Boundary conditions at the inlet area correspond to conditions of experimental measurement which were $20^{\circ} \mathrm{C}$ and atmospheric pressure.

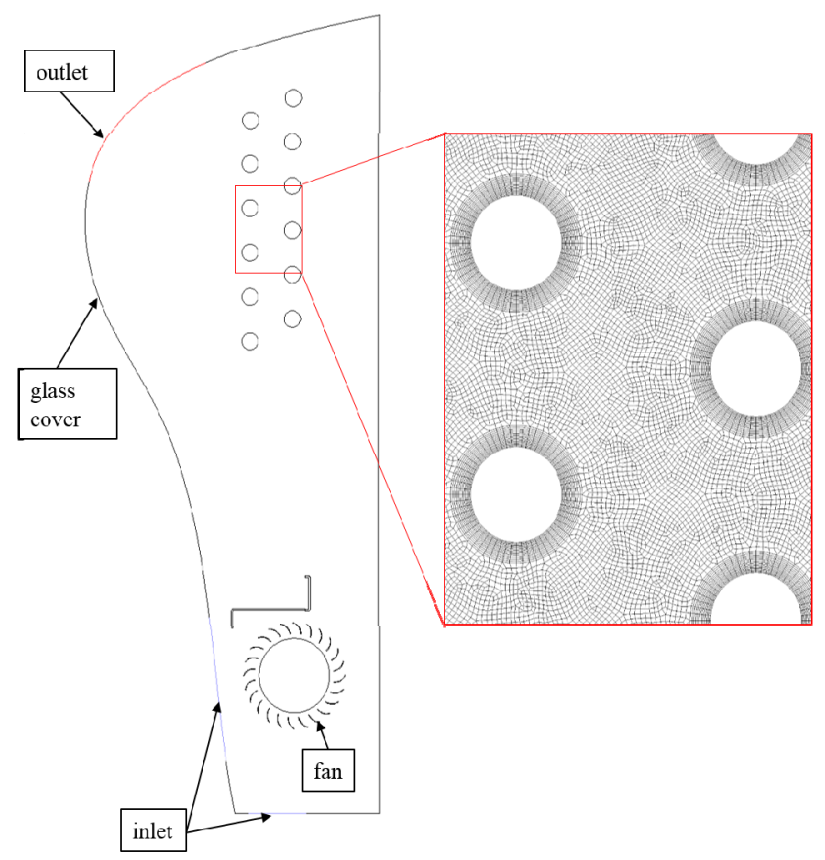

Figure 7. Two-dimensional computational domain (left), detail of computational mesh in vicinity of tubes (right).

Two-dimensional air flow was modelled by standard k- $\varepsilon$ turbulence model which was first introduced by Launder and Spalding [14]. Standard k- $\varepsilon$ turbulence model consists of two transport equations for turbulence kinetic energy $\mathrm{k}$ and its dissipation rate $\varepsilon$.

$$
\begin{aligned}
& \frac{\partial(\rho k)}{\partial t}+\frac{\partial\left(\rho k u_{i}\right)}{\partial x_{j}}=\tau_{i j} \frac{\partial\left(\rho u_{i}\right)}{\partial x_{j}}+\frac{\partial}{\partial x_{j}}\left[\left(\mu+\frac{\mu_{t}}{\sigma_{k}}\right) \frac{\partial k}{\partial x_{j}}\right]-\rho \varepsilon \\
& \frac{\partial(\rho \varepsilon)}{\partial t}+\frac{\partial\left(\rho \varepsilon u_{i}\right)}{\partial x_{j}}= \\
& =\frac{\varepsilon}{k} C_{\varepsilon 1} \tau_{i j} \frac{\partial\left(\rho u_{i}\right)}{\partial x_{j}}+\frac{\partial}{\partial x_{j}}\left[\left(\mu+\frac{\mu_{t}}{\sigma_{\varepsilon}}\right) \frac{\partial \varepsilon}{\partial x_{j}}\right]-\frac{\varepsilon^{2}}{k} C_{\varepsilon 2} \rho
\end{aligned}
$$

where $\rho$ is fluid density, $\mathbf{u}_{\mathrm{i}}$ are the components of velocity vector, $\mathbf{x}_{\mathbf{j}}$ are components of position vector, $t$ is time, $\boldsymbol{\tau}_{\mathbf{i j}}$ is Reynolds stress tensor, $\mu$ is dynamic viscosity and $\mu_{t}$ is turbulence eddy viscosity defined as

$$
\mu_{t}=C_{\mu} \rho \frac{k^{2}}{\varepsilon},
$$

where $\mathrm{C}_{\varepsilon 1}=1,44 ; \mathrm{C}_{\varepsilon 2}=1,92 ; \mathrm{C}_{\mu}=0,09 ; \sigma_{\mathrm{k}}=1 ; \sigma_{\varepsilon}=1,3$ are empirically derived constants which could be set by testing of simple fluid flow problems or by numerical optimization.

For the spatial discretization of computational domain the finite volume method is implemented in computational software Fluent. The second order upwind scheme was used to discretize convective terms of the governing equations. In ANSYS Fluent, the fluid flow in rotating domain could be solved using the multiple reference frame (MRF) function. This method is useful for the problems where some parts of geometry are moving (fan) and other parts are stationary (cover and walls of conductor). The main advantage of using MRF is, that there is no need to use any deforming mesh algorithm. The computational domain was divided by a circular interface into the two parts - the annular domain which contains fan blades and domain of surrounding fluid, see Fig. 8.

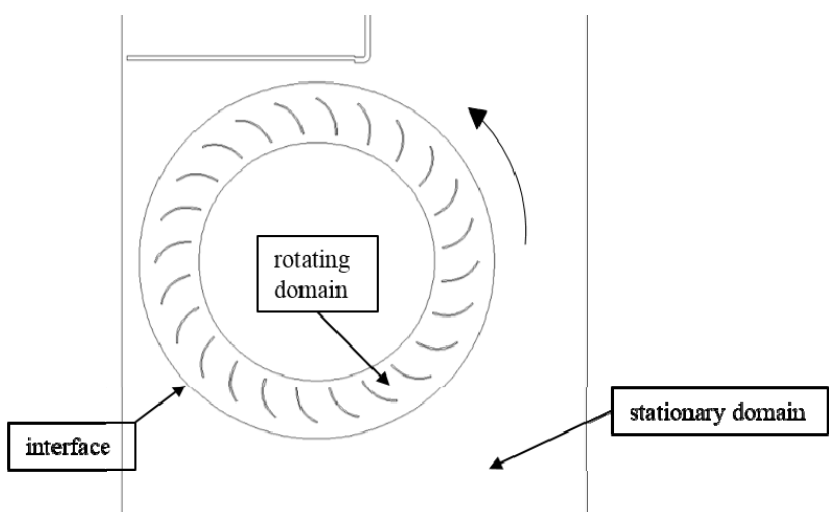

Figure 8. Division of domain into the moving and stationary part.

The blades are rotating with an angular velocity which is prescribed to whole rotating domain as a cell-zone condition in Fluent. Relative velocity must be applied into a numerical model to solve the fluid flow in rotating domain. This velocity is computed as Eq. (5).

$$
\vec{v}_{r}=\vec{v}-(\vec{\omega} \times \vec{r}),
$$

where $\vec{v}$ is the vector of absolute velocity, $\vec{\omega}$ is the angular velocity of rotating frame, $\vec{r}$ is the position vector in rotating frame.

\subsection{Results of numerical simulation}

Numerical simulations were provided for two fan velocities: 1000 and $1600 \mathrm{rpm}$. The comparison of convergence of solved cases is shown in the next Table 1 .

Table 1. The comparison of solved cases

\begin{tabular}{|l|c|c|c|c|}
\hline case & $\begin{array}{c}\text { number } \\
\text { of mesh } \\
\text { elements }\end{array}$ & rpm & $\begin{array}{c}\text { number of } \\
\text { iterations }\end{array}$ & $\begin{array}{c}\text { continuity } \\
\text { residual } \\
\text { value }\end{array}$ \\
\hline $\begin{array}{l}\text { Standard } \\
\text { shape }\end{array}$ & 210000 & 1000 & $10^{4}$ & $2,2 \cdot 10^{-8}$ \\
\cline { 3 - 5 } $\begin{array}{l}\text { New } \\
\text { shape }\end{array}$ & 250000 & 1600 & $10^{4}$ & $5,8 \cdot 10^{-7}$ \\
\cline { 3 - 5 } & & 1600 & $10^{4}$ & $2,9 \cdot 10^{-11}$ \\
\hline
\end{tabular}

Velocity vectors in computational domain in case of conventional steel cover are shown in the Fig. 9. 
Temperature distribution in computational domain for the first case of fan velocity is shown in the Fig. 10.
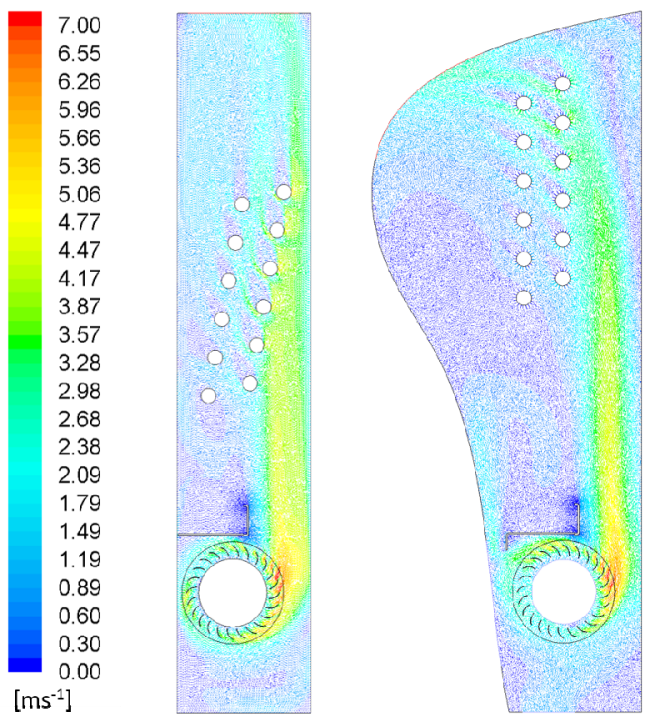

Figure 9. Velocity vectors for the $1000 \mathrm{rpm}$ fan velocity with the convetional steel cover in comparison to the new one.
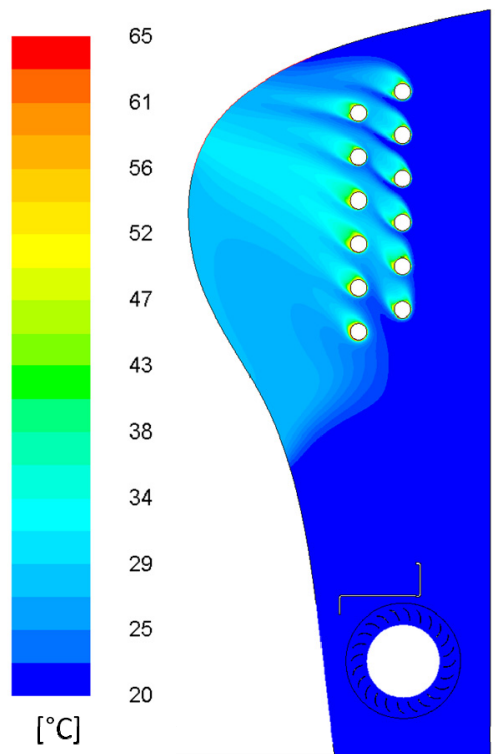

Figue 10. Temperature distribution for the $1000 \mathrm{rpm}$ fan velocity with inovated shape of cover.

The heat output was computed for each of these cases by formula

$$
P=Q c_{P} \Delta T,
$$

where $Q$ is the mass flow of air at the domain outlet, $\mathrm{c}_{\mathrm{p}}$ is specific heat equal to value $1006,43 \mathrm{Jkg}^{-1} \mathrm{~K}^{-1}$ and $\Delta \mathrm{T}$ is temperature difference between outlet and inlet area. The comparison of solved cases is shown in the Tab. 2 below.
Table 2. Heat output comparison

\begin{tabular}{|c|c|c|c|c|}
\hline case & $\mathrm{RPM}$ & $\mathrm{Q}\left[\mathrm{kg} \mathrm{s}^{-1}\right]$ & $\Delta \mathrm{T}\left[{ }^{\circ} \mathrm{C}\right]$ & $\mathrm{P}[\mathrm{W}]$ \\
\hline \multirow{2}{*}{$\begin{array}{c}\text { Standard } \\
\text { shape }\end{array}$} & 1000 & 0,186 & 6,8 & 1273 \\
\cline { 2 - 5 } & 1600 & 0,260 & 5,9 & 1544 \\
\hline \multirow{2}{*}{$\begin{array}{c}\text { New } \\
\text { shape }\end{array}$} & 1000 & 0,198 & 7,2 & 1435 \\
\cline { 2 - 5 } & 1600 & 0,271 & 6,1 & 1664 \\
\hline
\end{tabular}

\section{Results and discussion}

The experimental and numerical determination of heat outputs was performed in order to find a dependence on the shape of used covering front plate. The used exchanger optimized for forced convection achieves without using any fans only a very small thermal power. These quite small values could be measured only a very inaccurate with significant statistical variances, especially with a lowest Re numbers of the flowing medium in the exchanger. After turning on the fan even at very low speeds, the heat output starts to increase almost exponentially. Using of exchanger just without covering seems to be less appropriate. This methods are in practice used quite rarely, especially in some special customer applications like e.g. between the multi windows and facade systems.

As has been possible to observe, the ordinary rectangular metal sheeting, its geometry and dimensions are designed to reach the almost maximum possible power. In only one configuration of the curved cover with an upper convexity, we have succeeded in approximately $8 \%$ increasing of the heat power, compared to the conventional one. It can be concluded that the sheeting, its shape and parameters play a significant role in the construction of heating systems and to the heat output they have a very significant impact. This all is necessary to consider in a design process of new devices. It was produced, constructed and subsequently experimentally test a variety of shapes. These include position of the heat exchanger, its angle relative to the rear side and of course the shape of the used cover itself. The Fig. 11 and 12 show several variants where it was possible to observe the most fundamental differences. Figure 11 shows results of experimental measurement and Figure 12 shows results of numerical simulations.

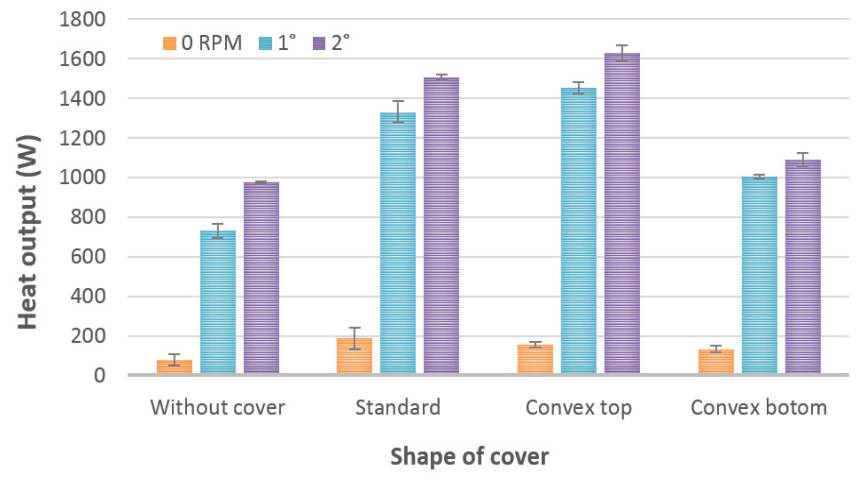

Figure 11. The selected measurement of heat outputs. 


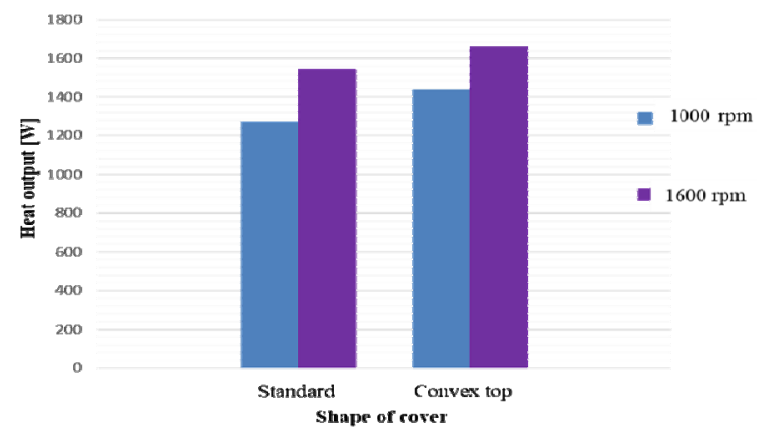

Figure 12. The simulation results of heat output dependence on angular velocity of the fan.

\section{Conlusion}

The differences between experimental and numerical results in values of heat output could be caused by simplification of the complex three-dimensional problem to a fluid flow in a two-dimensional domain. On the other hand, the simplified model was adequate to show the differences between standard and new glass cover. While considering the conventional shapes is in this solution achieved practically the maximal possible power. For consideration and as a subject for further work can be also clearly established the dependence of the heating medium and air flow velocity to the total heat output.

\section{Acknowledgements}

We give our thanks to the construction department and management of the company Licon heat s.r.o. for the collaboration and provided information.

This work was supported by the Ministry of Education of the Czech Republic within the SGS project no. 21007 on the Technical University of Liberec.

\section{References}

1. P. Kulhavy, M. Petru, P. Srb, G. Rachitsky: Eur. Phys. J. 92, 02064 (2014)

2. K. Frana, M. Muller, F. Lemfeld: WAS, Engineering and Technology 6, 5154 (2012)

3. Z. Chen, H. Guanren, M. Yuanle: Soc. for Industrial and Applied Math., xxix, 531 (2006)

4. LICON HEAT s.r.o., Směrnice technologie a konstrukce, Liberec (in Czech), (2014)

5. A. Hamza, G. M. Morsy, Energy efficiency and indoor thermal perception, 3 (2010)

6. T. Aicher, H. Martin, Intern. Journal of Heat and Mass Transfer, 40, 3617-3626 (1997)

7. T. Kuppan, Heat Exchangers Design Handbook, Taylor \& Francis, New York (2000)

8. P. Dancova, Z. Travnicek, T. Vit, EPJ Web of Conferences 45, 01002 (2013)

9. F. Lemfeld, K. Frana, The influence of geometry changes to the flow in a floor heating convector In 30th conf. of Dept of Fluid Mechanic, Liberec (2011)

10. F. Lemfeld, K. Frana, Journal of Applied Science in the Thermodynamics, 5 (2011)

11. EN $442-1$ ed. 2 Radiators and convectors: Technical specifications and requirements
12. M. Petru, O. Novak, D. Herak, S. Simanjuntak: Biosystems Eng., 111 (2012)

13. M. Petrů, O. Novák, L. Ševčík, P. Lepšík: Applied Mechanics and Materials, 157 (2014)

14. B. E. Launder, D. B. Spalding: Mathematical Models of Turbulence, Academic Press (1972) 